\section{A common genetic variation near INSIG2 is associated with obesity}

Around $10 \%$ of people carry a single-nucleotide polymorphism (SNP) that is associated with obesity, say American and German researchers.

Herbert et al. performed genome-wide screening of DNA samples from 694 Framingham study participants to identify SNPs potentially related to obesity. After comparing parent and offspring genotypes and phenotypes, only one SNP (rs7566605) had a statistically significant association with BMI.

The authors confirmed the robustness of the association between rs7566605 and BMI by analyzing previously published data for this SNP from a total of 9,881 participants in five additional multinational studies. The association was present in four of the five study populations, but no link between rs7566605 and BMI was found in the Nurses Health Study cohort, possibly because the study group comprised few individuals with a high BMI. Meta-analysis of case-control genotype data showed that individuals with a CC genotype at rs7566605 were $22 \%$ more likely to be obese than those with a GC or GG genotype.

The high prevalence of the rs7566605 $\mathrm{C}$ allele, and its distribution in Western European and African American individuals, prompted the authors to speculate that the $\mathrm{C}$ allele predates human migration from Africa, and was not always harmful. The proximity of rs 756660 to INSIG2 (insulin-induced gene 2) might explain its association with obesity: INSIG2 inhibits cholesterol and fatty-acid synthesis, and changes in its activity could elevate plasma triglyceride levels, and perhaps cause increased fat deposition.

Original article Herbert A et al. (2006) A common genetic variant is associated with adult and childhood obesity. Science 312: $279-283$

\section{Overdiagnosis is responsible for the increasing incidence of thyroid cancer in the US}

The incidence of thyroid cancer in the US is rising steeply, but it is unclear whether the increase is an artifact of increased scrutiny or altered diagnostic criteria, or whether the disease is occurring more frequently. To clarify the situation, Davies and Welch have examined trends relating to thyroid cancer in the US from 1973 to 2002.

Data from the Surveillance, Epidemiology, and End Results (SEER) program were used to calculate annual age-adjusted incidence and mortality rates for thyroid cancer (categorized as papillary, follicular, medullary, or anaplastic).

Between 1973 and 2002, the incidence of thyroid cancer more than doubled-from 3.6 to 8.7 cases per 100,000 people; however, this increase was almost entirely accounted for by the near-threefold increase in papillary tumors. Between 1988 and 2002, when tumor-size data were available, $\sim 87 \%$ of the 'excess' papillary cancers were $<2 \mathrm{~cm}$ in size; diagnostic criteria for this cancer did not change in this time.

The authors note that thyroid cancer mortality remained stable over the study period and conclude that the rising incidence has been caused by papillary thyroid cancer 'overdiagnosis'-increased detection of subclinical cancers (which often never become symptomatic and are a common autopsy finding) with advanced diagnostic and imaging techniques. Given the risk of complications associated with total thyroidectomy, and the likelihood that the number of thyroid cancers detected in the future will continue to rise, the authors recommend studies that explore the feasibility of a more cautious treatment strategy, perhaps one that includes watchful waiting.

Original article Davies L and Welch HG (2006) Increasing incidence of thyroid cancer in the United States, 1973-2002. JAMA 295: 2164-2167

\section{Bariatric surgery outperforms medical therapy for mild or moderate obesity}

The laparoscopic adjustable gastric band is a new bariatric surgical option for treatment of obese patients. This procedure has been proven to be safe and effective, is reversible, and requires only one overnight stay in hospital. Previously, few studies have compared surgical and medical therapies to treat obesity; O'Brien and colleagues, therefore, conducted a randomized, controlled trial to compare the effectiveness of the laparoscopic adjustable gastric band with that of an intensive, medical weight-loss program. 